\title{
Editorial Brain Tumor Immunotherapy
}

One emerging strategy in the treatment of tumors involves stimulation of an immunologic response against the neoplastic cells. The hope is that the immune system can be called into play to destroy malignant cells. However, in most instances, proliferating tumors do not provoke anti-tumor cellular immune responses. The precise mechanisms that enable antigenic neoplasms to escape host immunity are incompletely understood. The cells appear to escape recognition by the immune system in spite of the fact that neoplastic cells form weakly immunogenic tumor associated antigens (TAAs).

Tumor cells may evade immune responses by losing expression of antigens or major histocompatiblity complex (MHC) molecules or by producing immunosuppressive cytokines. A variety of strategies have been used to increase the immunogenetic properties of vaccine therapies for brain tumors. The immune response can be augmented by genetic modification of tumor cells to secrete cytokines including IL-2, GM-CSF and interferon- $\gamma$. One can also alter the MHC of the tumor cells to express allogeneic determinants. Finally one can genetically modify the tumor cells to express co-stimulatory molecules such as B7. In some instances, objective evidence of tumor regression has been observed in patients receiving immunizations only with tumor cell immunogens, suggesting the potential effectiveness of this type of immunotherapy for malignant neoplasms. In addition, modification of delivery techniques to treat intracerebral tumors has included intrathecal, intralymphatic, subcutaneous and intratumoral injections of treatment cells. An alternative strategy is to genetically modify tumor cells to express an antisense gene to TGF- $\beta$, which is a cytokine highly expressed in glioma cells that acts to inhibit the function of cytotoxic T cells.

Previous immunotherapy strategies have also utilized classical immunologic cell types including activated lymphocytes and LAK cells. More recently, a variety of cells have been investigatped for their usefulness in tumor oncology including tumor cells themselves (syngeneic or allogeneic), dendritic cells or fibroblasts (syngeneic or allogeneic). Although syngeneic tumor cells have the advantage that they express most of the appropriate antigens needed for targeted therapy, many types of tumors are difficult to establish in culture. In addition, cytokine gene therapies requiring the transduction of autologous tumor cells may not be practical for many cancer patients. Modification of neoplastic cells taken directly from tumor-bearing patients may be difficult. In particular a primary tumor cell line, required for retroviral modification has to be established. An alternative cell type that can be used for therapeutic immunizations is the dendritic cell (DC), which is a specialized antigen presenting cell. Pre-clinical studies have indicated that immunizing either mice or rats with DC pulsed using tumor cell antigens can stimulate a cytotoxic $\mathrm{T}$ cell response that is tumorspecific and that engenders protective immunity against CNS tumor in the treated animals.

The ultimate goal of cancer therapy is the elimination of every remaining tumor cell from the patient. It is unlikely that a single form of therapy is capable of achieving this goal. However immunotherapy in combination with surgery, radiation therapy and chemotherapy will likely find a place as a new and important means of treatment for patients with brain tumors. In this special issue papers are provided exploring the potential of different immunotherapeutic strategies for the treatment of brain tumors along with related aspects of tumor immunotherapy. In particular there is a paper describing the development of a unique DNA-based vaccine which is effective in animal models for treatment of intracerebral tumors. A second paper is included which provides data indicating that animals receiving such therapeutic vaccines failed to exhibit signs of autoimmunity, and tumors derived from the vaccine itself failed to develop in immune-competent tumor-free mice. These are important safety considerations to be considered upon the application of tumor vaccines. Also included is a paper that explores the mechanisms of cytokine-induced immunosuppression which is critical in developing improvements in anti-tumor vaccines. Another paper is provided that describes therapeutic strategies that target stem cell associated molecules that have been isolated from brain tumor tissue, and immunotherapy targets at brain tumor stem cells is explored. Finally a unique study is included exploring some of the metabolic and immunotherapeutic effects of thiazodinedione agonists, which have been shown to exhibit anti-tumor effects in general and have also been shown to have some efficacy in the treatment of gliomas.

Terry Lichtor (Guest Editor)

Department of Neurological Surgery Rush University Medical Center 1725 West Harrison Street, Suite 1115 Chicago, Illinois 60612, USA

Telephone: 312-942-6628

E-mail: Terry_Lichtor@rush.edu

(C) Terry Lichtor; Licensee Bentham Open.

This is an open access article licensed under the terms of the Creative Commons Attribution Non-Commercial License (http://creativecommons.org/ licenses/by-nc/3.0/) which permits unrestricted, non-commercial use, distribution and reproduction in any medium, provided the work is properly cited. 